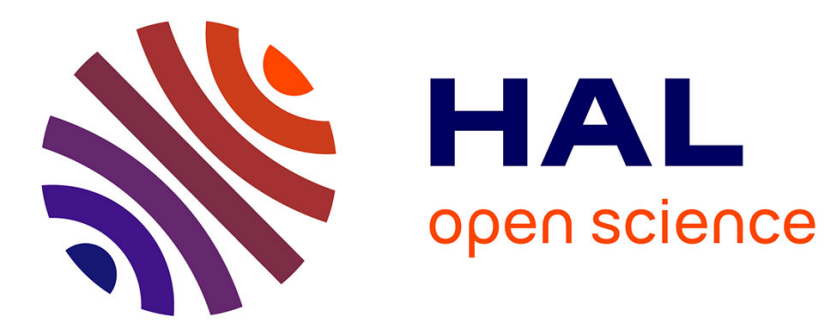

\title{
Measurement of the lifetime of the $\tau$ lepton
}

M. Acciarri, O. Adriani, M. Aguilar-Benitez, S. Ahlen, B. Alpat, J. Alcaraz, G. Alemanni, J. Allaby, A. Aloisio, G. Alverson, et al.

\section{To cite this version:}

M. Acciarri, O. Adriani, M. Aguilar-Benitez, S. Ahlen, B. Alpat, et al.. Measurement of the lifetime of the $\tau$ lepton. Physics Letters B, 1996, 389, pp.187-196. 10.1016/j.nuclphysbps.2015.02.007 . in2p300001181

\section{HAL Id: in2p3-00001181 https://hal.in2p3.fr/in2p3-00001181}

Submitted on 11 Feb 1999

HAL is a multi-disciplinary open access archive for the deposit and dissemination of scientific research documents, whether they are published or not. The documents may come from teaching and research institutions in France or abroad, or from public or private research centers.
L'archive ouverte pluridisciplinaire HAL, est destinée au dépôt et à la diffusion de documents scientifiques de niveau recherche, publiés ou non, émanant des établissements d'enseignement et de recherche français ou étrangers, des laboratoires publics ou privés. 
EUROPEAN ORGANIZATION FOR NUCLEAR RESEARCH

CERN-PPE/96-124

September 13, 1996

\title{
Measurement of the Lifetime of the Tau Lepton
}

\author{
The L3 Collaboration
}

\begin{abstract}
The lifetime of the tau lepton is measured using data collected in 1994 by the L3 detector at LEP. The precise track position information of the Silicon Microvertex Detector is exploited. The tau lepton lifetime is determined from the signed impact parameter distribution for 30322 tau decays into one charged particle and from the decay length distribution for 3891 tau decays into three charged particles. Combining the two methods we obtain $\tau_{\tau}=290.1 \pm 4.0 \mathrm{fs}$.
\end{abstract}

Submitted to Phys. Lett. B 


\section{Introduction}

In the Standard Model of electroweak interactions [1], the couplings of charged and neutral currents to leptons are independent of the lepton generation. The most basic test of this universality for the charged current is the measurement of the coupling strength of electron, muon and tau to the $\mathrm{W}$ boson. The vertex factor for tau decays and muon decays is the same under the hypothesis of lepton universality in charged currents, thus their leptonic widths should be related by a simple phase-space factor. The leptonic tau decay rate [2]

$$
\begin{aligned}
\Gamma\left(\tau^{-} \rightarrow \ell^{-} \bar{\nu}_{\ell} \nu_{\tau}\right) & \equiv \frac{\mathrm{BR}\left(\tau^{-} \rightarrow \ell^{-} \bar{\nu}_{\ell} \nu_{\tau}\right)}{\tau_{\tau}} \\
& =\frac{G_{F}^{2} m_{\tau}^{5}}{192 \pi^{3}}\left(1+\delta_{m}\right)\left(1+\delta_{W}\right)\left(1+\delta_{\text {rad }}\right) \quad \ell^{-}=\mathbf{e}^{-}, \mu^{-}
\end{aligned}
$$

gives the total coupling strength, measured by the Fermi constant $G_{F}$ which is defined by the analogous relation for muon decays. The correction $\delta_{m}$ takes into account the finite mass of the charged lepton in the final state. The $\mathrm{W}$ propagator effects lead to the correction $\delta_{W}$, while QED radiative corrections are introduced by the last factor, $\delta_{\text {rad }}$. Therefore a measurement of the tau lifetime $\left(\tau_{\tau}\right)$ and leptonic branching ratios provides a test of lepton universality in charged weak currents.

In this paper, we present a measurement of the tau lifetime using two different methods. In the first method, the lifetime is extracted from the observed decay length distribution in threeprong tau decays. The second method uses the impact parameter distribution in one-prong tau decays. This new measurement makes use of the enhanced tracking capabilities of the Silicon Microvertex Detector [3] and increased statistics.

\section{The L3 Detector}

The L3 detector [4] consists of a central tracking system, a fine-grained electromagnetic calorimeter composed of BGO crystals, a ring of plastic scintillation counters, a hadron calorimeter with uranium absorber and proportional wire chambers, and a muon spectrometer consisting of multi-wire drift chambers. These detectors are installed in a $12 \mathrm{~m}$ diameter solenoidal magnet which provides a magnetic field of $0.5 \mathrm{~T}$ along the beam direction.

The measurement is based on the information obtained from the central tracking system, which is composed of a Silicon Microvertex Detector (SMD), a Time Expansion Chamber (TEC) and a Z-chamber. The SMD is made of two concentric layers of double-sided silicon detectors, placed at about 6 and $8 \mathrm{~cm}$ from the beam line. Each layer provides a two-dimensional position measurement, with a resolution of 7 and $14 \mu \mathrm{m}[5,6]$ for normal incident tracks, in the directions perpendicular and parallel to the beam direction, respectively. The TEC consists of two coaxial cylindrical drift chambers with 12 inner and 24 outer sectors. The sensitive region is between 10 and $45 \mathrm{~cm}$ in the radial direction, with 62 layers of wires giving a spatial resolution of approximately $50 \mu \mathrm{m}$ in the plane perpendicular to the beam axis ( $\mathrm{r} \phi$ plane).

\section{Selection of $\mathbf{Z} \rightarrow \tau^{+} \tau^{-}$Decays}

For this analysis data collected in 1994 by the L3 detector are used, which correspond to an integrated luminosity of $46 \mathrm{pb}^{-1}$. The strategy for selecting $\mathrm{Z}$ decays into tau pairs is to identify 
events which contain two highly collimated, back-to-back and low-multiplicity jets. For this purpose the track multiplicity, the jet energy and the jet direction are used.

\subsection{Event Selection}

In order to have high-quality reconstruction of the tracks, $\mathrm{Z}$ decays into $\tau^{+} \tau^{-}$pairs are selected within a fiducial volume defined by $\left|\cos \theta_{t}\right|<0.72$ (barrel region), where the polar angle $\theta_{t}$ is given by the thrust axis of the event. The event is required to have at least two jets, corresponding to the two taus, and the energy of the most energetic jet is required to be greater than $8 \mathrm{GeV}$. The acollinearity angle between the directions of the two highest-energy jets must be smaller than $10^{\circ}$ to reduce the background from two-photon processes.

In order to remove hadronic $\mathrm{Z}$ decays, the number of tracks in each jet must be less than four. In addition the maximum angle between the track direction and the thrust axis of the nearest jet must be less than $18^{\circ}$ and $22^{\circ}$, for the most energetic and the second most energetic jets, respectively.

The background from $\mathrm{e}^{+} \mathrm{e}^{-} \rightarrow \mathrm{e}^{+} \mathrm{e}^{-}(\gamma)$ is reduced by requiring the total energy deposited in the electromagnetic calorimeter to be less than $75 \%$ of the center-of-mass energy. In addition, the two highest-energy clusters in the electromagnetic calorimeter having an electromagnetic shower shape, must have energies below $85 \%$ and $80 \%$ of the beam energy.

In order to reject $\mathrm{e}^{+} \mathrm{e}^{-} \rightarrow \mu^{+} \mu^{-}(\gamma)$ events, the total momentum measured in the muon spectrometer must be less than $70 \%$ of the center-of-mass energy. In addition, if the momentum of a muon exceeds $80 \%$ of the energy of the associated jet (isolated muon), this momentum is required to be less than $80 \%$ of the beam energy. If muons are not reconstructed in the muon chambers they can be identified by a minimum ionizing energy deposit. Therefore, in events with an energy in the hadronic calorimeter of less than $7 \mathrm{GeV}$, we require a track length in this calorimeter of less than $23 \mathrm{~cm}$ for at least one jet. This rejects dimuon events, as well as cosmic-ray events. The cosmic-ray background is further reduced by requiring a scintillation counter hit within $5 \mathrm{~ns}$ of the beam crossing. In addition, the distance of closest approach to the interaction point measured in the muon chambers must be less than two standard deviations of the resolution.

Following this procedure, 31108 events are selected in the barrel region from the data collected in 1994. The selection efficiency in the fiducial volume is determined to be $77.7 \%$ from $\mathrm{e}^{+} \mathrm{e}^{-} \rightarrow \tau^{+} \tau^{-}(\gamma)$ Monte Carlo events generated with KORALZ [7] and simulated with GEANT [8]. In the selected sample a background of $0.53 \%, 0.58 \%$ and $0.22 \%$, determined by Monte Carlo, remains from $\mathrm{Z}$ decays into electrons, muons and hadrons, respectively. The contamination of cosmic rays and two-photon processes are estimated to be $0.1 \%$ each. The relative uncertainty on the background fractions is approximately $20 \%$.

\subsection{Selection of One- and Three- Prong Tau Decays}

In addition to the selection of $\mathrm{Z} \rightarrow \tau^{+} \tau^{-}$decays, cuts are applied to the tracks in order to ensure a good quality of the reconstruction. There must be at least 40 TEC hits on each track and at least one hit from the SMD inner $r \phi$ layer. The transverse momentum of the track must be greater than $500 \mathrm{MeV}$ and the impact parameter, $\delta$, must be less than $10 \mathrm{~mm}$.

The event is divided into two hemispheres by the plane perpendicular to the thrust axis. Hemispheres with only one track are selected for the impact parameter method and those with three tracks are selected for the decay length method. For the three prong candidates, only 
two of the three tracks are required to have matched SMD hits. In addition the $\chi^{2}$ probability of the secondary vertex is required to be larger than $1 \%$.

\section{Tracking Performance}

In both methods used in this analysis it is crucial to have a systematically accurate determination of the impact parameter and also of its error. The experimental resolutions on the impact parameter and the decay length are related to the error on the impact parameter; therefore a track-by-track knowledge of this error is required. An estimator of the intrinsic impact parameter error is given by the track fit error, $\sigma_{f i t_{i}}$. In addition to this, uncertainties from the horizontal and vertical size of the interaction region, $\sigma_{x, y}$, and from the multiple scattering, $\sigma_{M S_{i}}$, have to be taken into account. The total impact parameter error for a certain track, $i$, is then

$$
\sigma_{\delta_{i}}^{2}=\sigma_{f i t_{i}}^{2}+\sigma_{x}^{2} \sin ^{2} \phi_{i}+\sigma_{y}^{2} \cos ^{2} \phi_{i}+\sigma_{M S_{i}}^{2}
$$

where $\phi_{i}$ is the track azimuthal angle.

The tau production point is estimated from the average beam position during a LEP fill, found from hadronic events. The size of the interaction region is determined from Bhabha and dimuon events to be $\sigma_{x}=119 \pm 1 \mu \mathrm{m}$ and $\sigma_{y}=12 \pm 2 \mu \mathrm{m}$ in the horizontal and vertical directions, respectively.

Figure 1 shows the distribution of the distance between the two tracks in the plane perpendicular to the beam axis (miss distance), for Bhabha and dimuon events. The solid line represents the result of a fit to the distribution with the sum of two Gaussian functions. An average impact parameter intrinsic resolution of $26 \mu \mathrm{m}$ can be estimated for $72 \%$ of the tracks. For the remaining tracks the average resolution is $61 \mu \mathrm{m}$.

The impact parameter resolution function, $R$, is described in terms of a double Gaussian function

$$
R\left(\delta, \delta_{i}, \sigma_{\delta_{i}}\right)=\frac{1}{\sqrt{2 \pi} \sigma_{\delta_{i}}}\left\{\frac{1-f}{\kappa_{1}} \exp \left[-\frac{1}{2}\left(\frac{\delta-\delta_{i}}{\kappa_{1} \sigma_{\delta_{i}}}\right)^{2}\right]+\frac{f}{\kappa_{2}} \exp \left[-\frac{1}{2}\left(\frac{\delta-\delta_{i}}{\kappa_{2} \sigma_{\delta_{i}}}\right)^{2}\right]\right\}
$$

where $f$ is the relative fraction of the second Gaussian and $\kappa_{1,2}$ are correction factors for the estimated total impact parameter error, $\sigma_{\delta_{i}}$. For the decay length measurement, a similar parametrization is used for the resolution function $R\left(L, L_{i}, \sigma_{L_{i}}\right)$. The parameters $f, \kappa_{1,2}$, allow for residual systematic effects in the tracking detectors, beam spot position and size, and the treatment of multiple scattering.

The quality of the description of the resolution function for the impact parameter is checked using a lifetime-free sample (Bhabha and dimuon events). Figure 2 shows the distribution of the ratio between the impact parameter and its uncertainty, as determined from the resolution function. This quantity is normally distributed with a width consistent with unity, reflecting a good description of the resolution function.

For tau decays, the values for $f$ and $\kappa_{1,2}$ are determined from the tau sample itself, in the lifetime fit procedure (see section 4 ). The results are shown in table 1 . 


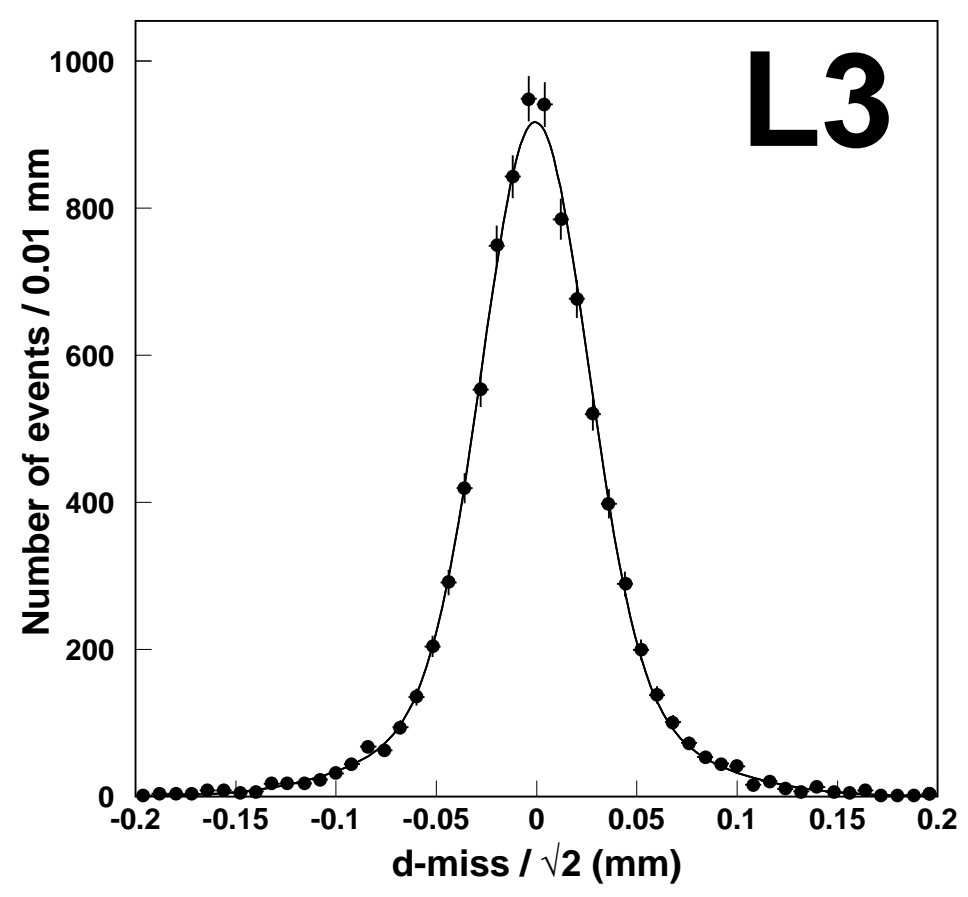

Figure 1: Distribution of the miss distance for Bhabha and dimuon events.

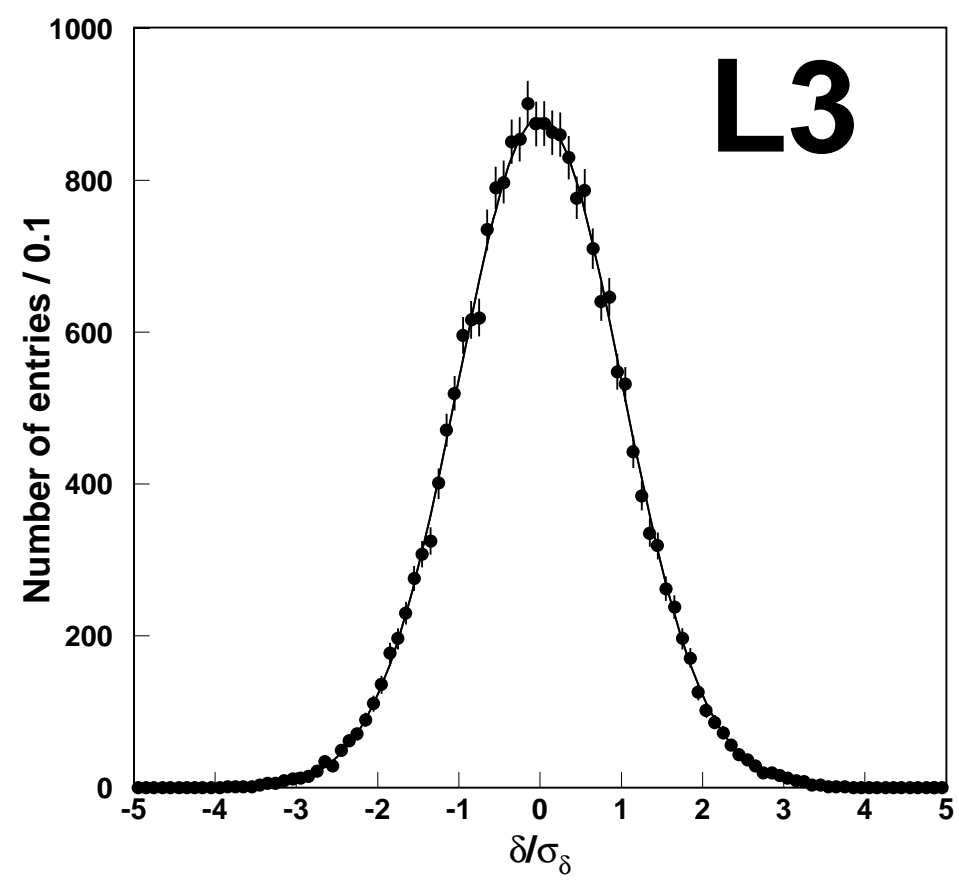

Figure 2: Distribution of the ratio between impact parameter and its uncertainty as determined from the resolution function, for Bhabha and dimuon events. Each track is entered twice, with a relative weight according to the two Gaussians. The line represents the result of a single Gaussian fit, which gives an average value of $-0.005 \pm 0.007$ and a sigma of $1.005 \pm 0.005$ 


\begin{tabular}{|l|c|c|c|}
\hline Method & $f(\%)$ & $\kappa_{1}$ & $\kappa_{2}$ \\
\hline \hline Impact Parameter & $19 \pm 4$ & $\mathbf{0 . 9 2} \pm \mathbf{0 . 0 2}$ & $1.8 \pm \mathbf{0 . 1}$ \\
Decay Length & $\mathbf{9 . 6 \pm 2 . 6}$ & $\mathbf{0 . 9 3} \pm \mathbf{0 . 0 4}$ & $2.2 \pm \mathbf{0 . 2}$ \\
\hline
\end{tabular}

Table 1: Parameters of the resolution functions for the impact parameter and the decay length methods.

\section{Lifetime Measurement}

The two techniques used to measure the tau lifetime are illustrated in figure 3 . The decay length of the tau (see figure 3a) is obtained from the secondary vertex reconstructed from three-prong tau decays. The tau decay point is found in the plane perpendicular to the beam axis and its distance, $L_{x y}$, from the beam position is determined from a $\chi^{2}$ minimization. The result is
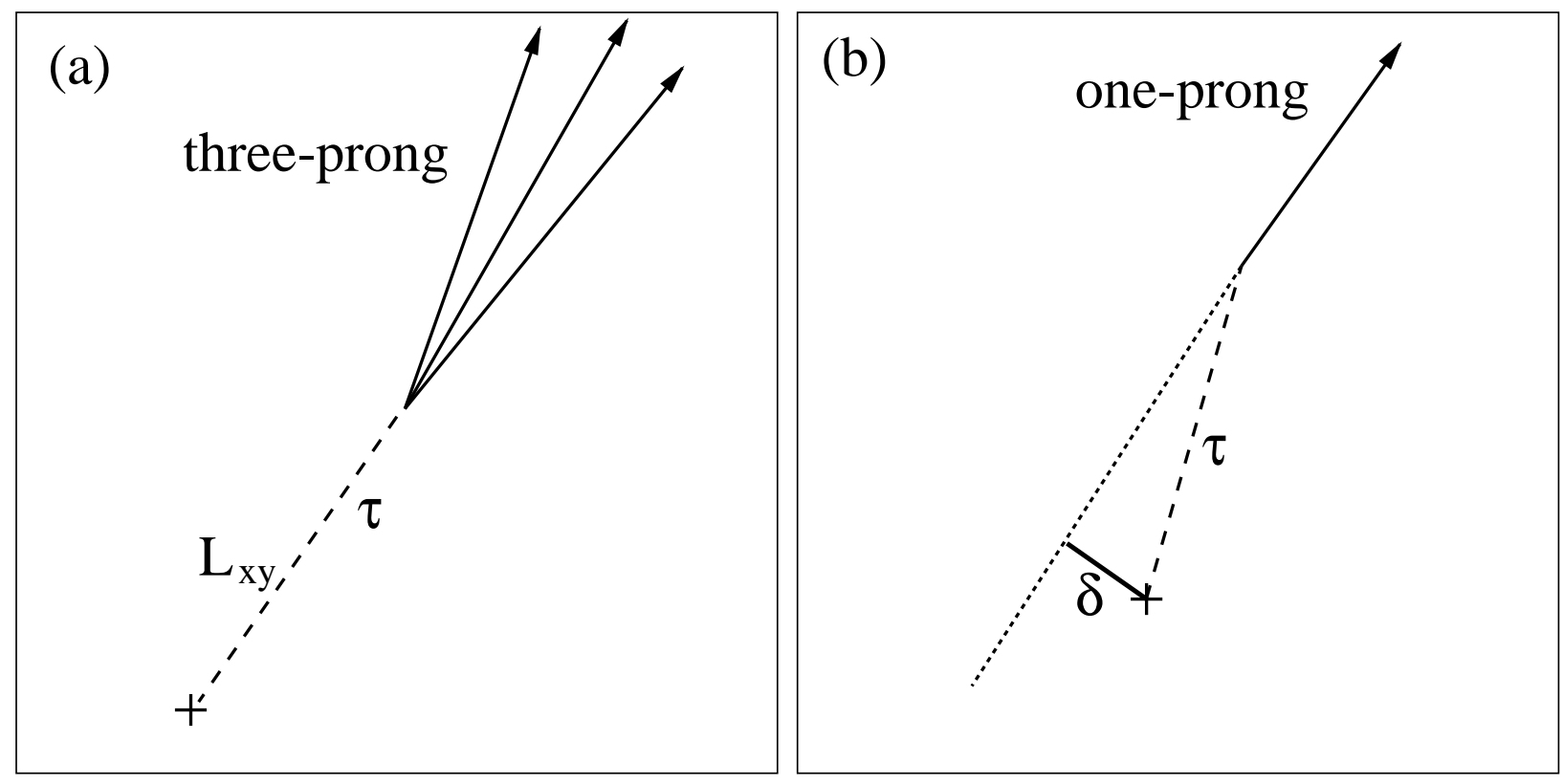

Figure 3: Schematic view of decay length (a) and impact parameter (b).

then converted into a three-dimensional decay length by means of the polar angle of the thrust axis. The average decay length, obtained from a maximum-likelihood fit, is translated into the tau lifetime, using the average boost of the tau, which takes into account energy losses due to radiation.

The impact parameter (see figure $3 \mathrm{~b}$ ) is the distance of closest approach, $\delta$, of the track to the tau production point, which is estimated by the beam position. In order to be sensitive to the lifetime, a sign is given to the impact parameter, according to the position of the intersection between the track and the tau direction of flight. The tau lifetime is extracted from a maximum-likelihood fit to the observed signed impact parameter distribution.

For both methods the likelihood function is expressed in terms of a convolution between an underlying physics function, $P$, and a detector resolution function, $R$ (see eq. 3). An additional term for the background fraction, $f_{B}$, is considered. The function $B$ is equal to the resolution function for the hadronic background in the three-prong sample and for the Bhabha 
and dimuon background in the one-prong sample, while it is a constant function for the cosmic muon background. The likelihood function is thus:

$$
\log \mathcal{L}\left(\tau_{\tau}, f, \kappa_{1}, \kappa_{2}\right)=\sum_{i=1}^{N} \log \left\{\left(1-f_{B}\right) P\left(\tau_{\tau}, \delta^{\prime}\right) \otimes R\left(\delta^{\prime}, \delta_{i}, \sigma_{i}\right)+f_{B} B\left(\delta_{i}, \sigma_{i}\right)\right\}
$$

\subsection{Decay Length Method}

Figure 4(a) shows the distribution of measured decay lengths of three-prong tau decays selected with the procedure described in section 2. A sample of 3891 three-prong tau decays, with a decay length in the range of $[-10,20] \mathrm{mm}$ and a calculated decay length error of less than $3 \mathrm{~mm}$ is used for the lifetime determination. The average tau decay length is extracted from an unbinned maximum-likelihood fit (see equation 4). The probability density function is given by the convolution between an exponential physics function and the resolution function with parameters as given in table 1 . The average tau decay length obtained from the fit is $\langle L\rangle=2.254 \pm 0.041 \mathrm{~mm}$, which is converted to a tau lifetime using the Lorentz boost factor.

The value for the tau lifetime from the decay length method is

$$
\tau_{\tau}=293.0 \pm 5.3 \mathrm{fs},
$$

where the error is statistical only. The solid line in figure 4(a) represents the result of this fit. The significance of the difference between the fit and the data points is shown in figure $4(\mathrm{~b})$. Good agreement between data and the fit result over the whole range of decay length values is observed.

In order to check for possible biases, the same procedure to obtain the tau lifetime is also applied to a Monte Carlo sample, generated with KORALZ. No significant deviation between the input tau lifetime and the result of the fit is observed, and a systematic error is conservatively assigned, equivalent to the statistical precision of this test. A systematic error due to the uncertainty in the resolution function is evaluated by varying the parameters of this function within their errors, taking into account correlations. The effect of the background uncertainty is checked both by a $20 \%$ relative variation of the background fraction and by leaving it free in the lifetime fit. The cuts on the decay length range, the decay length error and the secondary vertex $\chi^{2}$, are varied by $50 \%$ to evaluate their contributions to the systematic error. The

\begin{tabular}{|l|c|}
\hline Source & Error (fs) \\
\hline \hline Fit bias & 1.5 \\
Resolution Function & 1.5 \\
Background uncertainty & $\mathbf{0 . 5}$ \\
Fit range & 1.2 \\
Secondary vertex cut & $\mathbf{0 . 5}$ \\
\hline
\end{tabular}

Table 2: Systematic errors for the decay length method.

contributions to the systematic error for this measurement are summarized in table 2 and are added in quadrature to obtain the total systematic error. The result for the tau lifetime from the decay length method is

$$
\tau_{\tau}=293.0 \pm 5.3(\text { stat }) \pm 2.5 \text { (syst) fs. }
$$




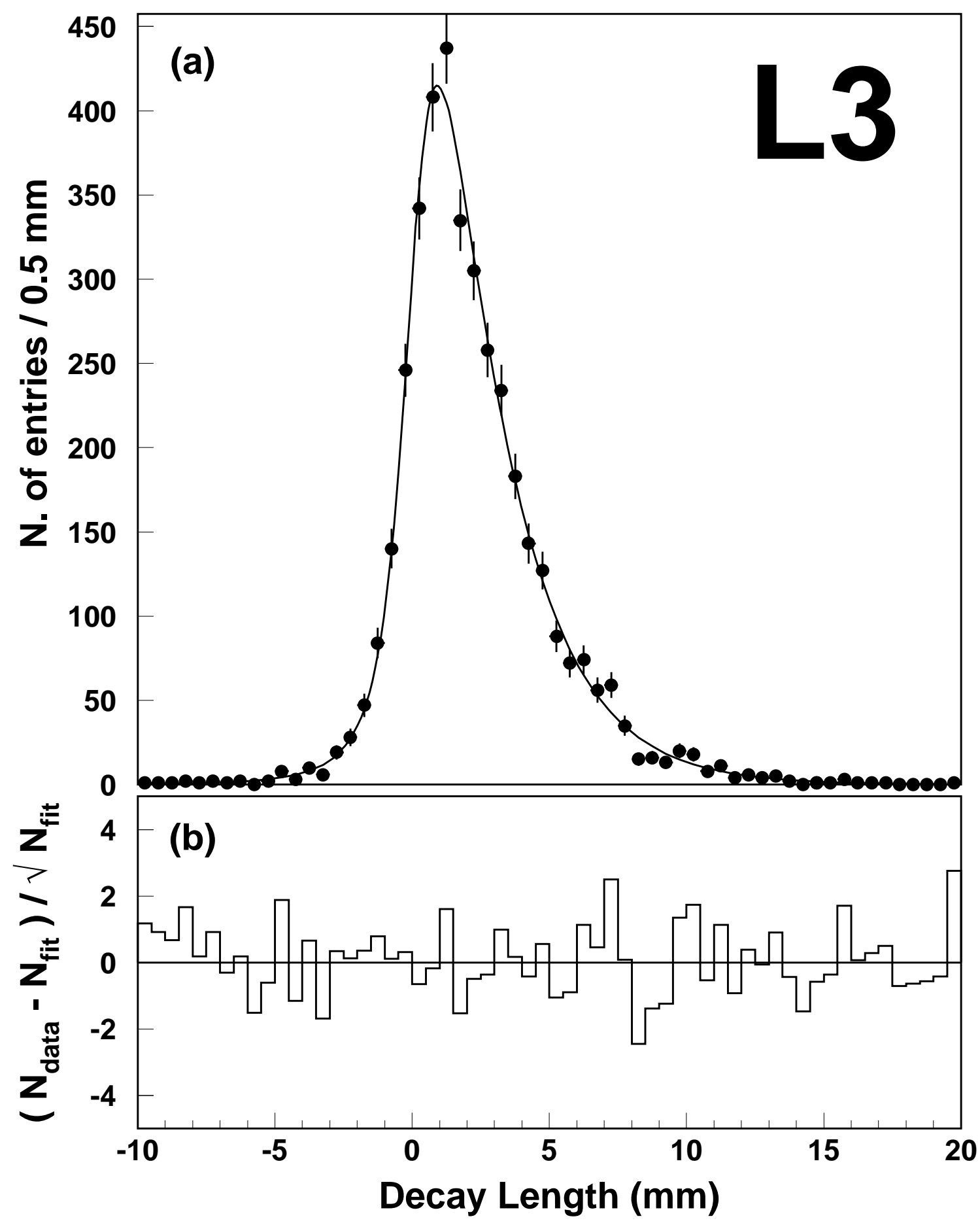

Figure 4: The decay length distribution for three-prong tau decays is shown in (a). The points are the data and the solid line is the result of the fit described in the text. Plot (b) shows the significance of the difference between the observed decay length distribution and the fit result. 


\subsection{Signed Impact Parameter Method}

The distribution of the measured signed impact parameter of one-prong tau decays, selected with the procedure described in section 2, is shown in figure 5(a). A sample of 30322 one prong tau decays with a signed impact parameter in the range of $[-1.0,1.5] \mathrm{mm}$ and a calculated error of less than $150 \mu \mathrm{m}$ is used for the lifetime determination. The tau lifetime is obtained from an unbinned maximum-likelihood fit to this distribution (see equation 4 . The probability density function for each track is given by the convolution of a physics function and the detector resolution function with parameters as given in table 1 . The physics function is determined from the signed impact parameter distribution of a Monte Carlo sample generated with KORALZ, for which the same selection procedure is applied as for data. The true impact parameter is used without the effect of resolution. However the physics function includes the possibility of wrong sign assignment due to the approximation of the tau direction by the reconstructed event thrust axis.

The value for the tau lifetime obtained from the signed impact parameter method is

$$
\tau_{\tau}=287.5 \pm 3.8 \mathrm{fs},
$$

where the error is statistical only. The solid line in figure 5(a) represents the result of the fit. Figure 5(b) shows the significance of the difference between the data points and the fit value. Good agreement between data and the fit result over the whole range of signed impact parameter values is observed.

In order to check for possible biases in the method, the lifetime fit is performed on the Monte Carlo sample, with the same procedure as used for data. No significant bias is observed and a systematic error is conservatively assigned, equal to the statistical precision of this test. The systematic effect due to the knowledge of the physics function is evaluated by taking into account its statistical uncertainty and its dependence on the tau lifetime in the range from 200 to $400 \mathrm{fs}$. A systematic error due to the resolution function is evaluated by varying the parameters of this function within their errors, taking into account correlations. As a check we evaluate the change in the tau lifetime when using the resolution function parameters obtained from the lifetime-free sample. The result is consistent within the systematic error assigned due to the uncertainties in the resolution function parameters. The uncertainty in the resolution function due to the knowledge of the beam spot size is evaluated by varying the beam spot size parameters by one standard deviation. The effect of the background uncertainty is checked using a $20 \%$ relative variation of the background fraction. The cuts on the range of the impact parameter and its error are varied by $50 \%$ to evaluate their contributions to the systematic error.

The contributions to the systematic error for this measurement are shown in table 3 . The tau lifetime obtained from the signed impact parameter method is

$$
\tau_{\tau}=287.5 \pm 3.8(\text { stat }) \pm 3.9 \text { (syst) fs. }
$$

\section{Summary and Conclusions}

The tau lepton lifetime is measured using the signed impact parameter and the decay length methods. The two results, which are consistent with one another, are independent since they are obtained using different sets of tau decays and the systematic errors are uncorrelated. 


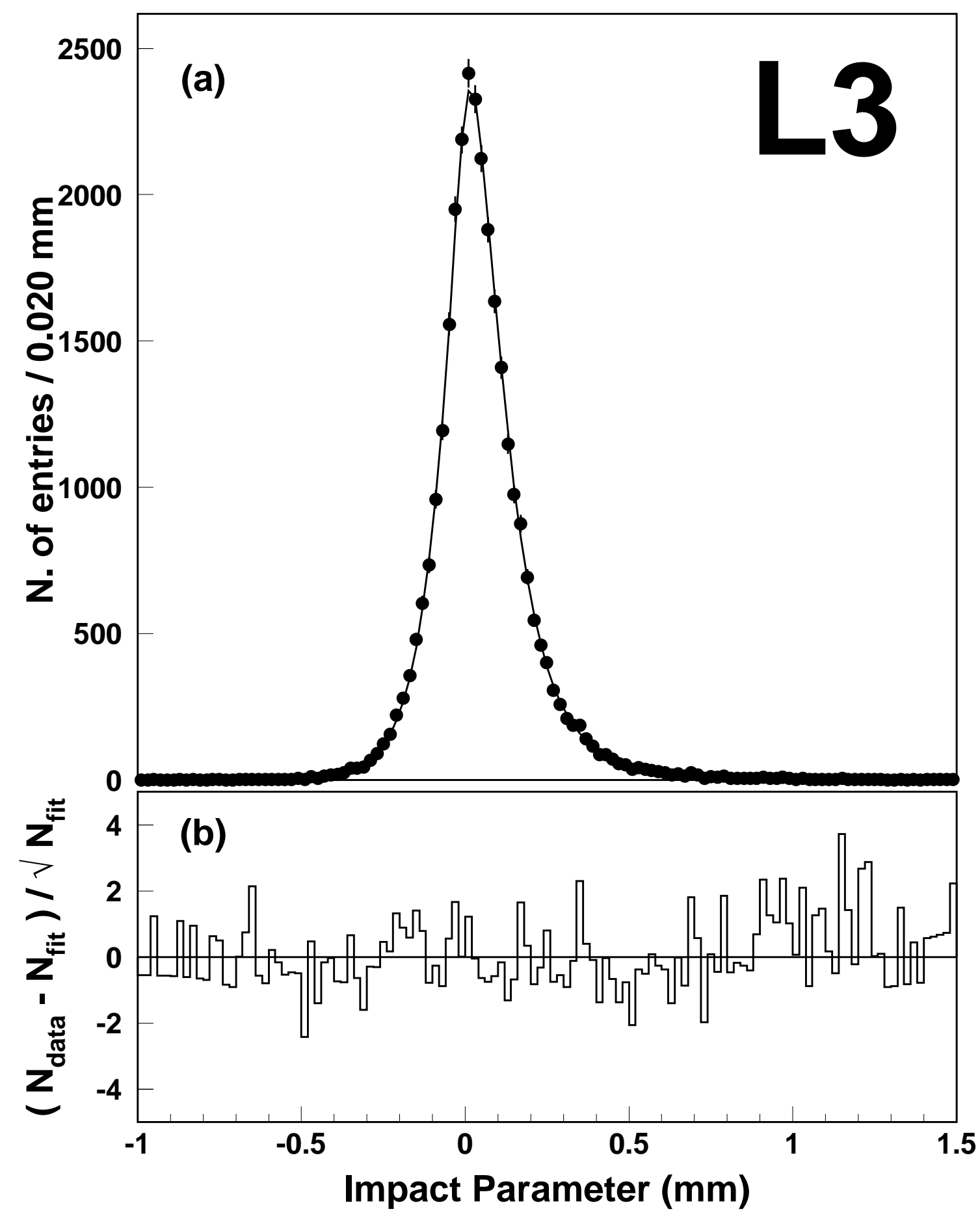

Figure 5: The signed impact parameter distribution for one-prong tau decays is shown in (a). The points are the data and the solid line is the result of the fit described in the text. Plot (b) shows the significance of the difference between the observed distribution and the fit result. 


\begin{tabular}{|l|c|}
\hline Source & Error (fs) \\
\hline \hline Fit bias & 1.5 \\
Physics Function & 2.2 \\
Resolution Function & 2.6 \\
Beam Spot Size & 0.5 \\
Background uncertainty & 1.0 \\
Fit range & 0.5 \\
\hline
\end{tabular}

Table 3: Systematic errors for the signed impact parameter method.

Combining the two results we obtain:

$$
\tau_{\tau}=290.1 \pm 4.0 \mathrm{fs}
$$

This result is in agreement with, and more precise than our previous measurements obtained with 1991-93 data $[9,10]$, and it is in agreement with the current world average value [11].

\section{Acknowledgments}

We wish to express our gratitude to the CERN accelerator divisions for the excellent performance of the LEP machine. We acknowledge the contributions of all the engineers and technicians who have participated in the construction and maintenance of this experiment. Those of us who are not from member states thank CERN for its hospitality and help.

\section{References}

[1] S. Glashow, Nucl. Phys. 22 (1961) 579;

S. Weinberg, Phys. Rev. Lett. 19 (1967) 1264;

A. Salam, in Elementary Particle Theory, N. Svartholm ed., Almqvist and Wiksell, Stockholm (1968) 367.

[2] W. Marciano and A. Sirlin, Phys. Rev. Lett. 61 (1988) 1815.

[3] M.Acciarri et al., Nucl. Inst. Meth. A 351 (1994) 300.

[4] L3 Collab., B. Adeva et al., Nucl. Inst. Meth. A 289 (1990) 35.

[5] A.Adam et al., Nucl. Inst. Meth. A 348 (1994) 436.

[6] A.Adam et al., Nucl. Inst. Meth. A 344 (1994) 521.

[7] S. Jadach, B.F.L. Ward and Z. Was, The Monte Carlo Program KORALZ, CERN preprint CERN-TH/5994/91 (1991); Comp. Phys. Comm. 66 (1991) 276.

[8] The L3 detector simulation is based on GEANT Version 3.15.

See R. Brun et al., "GEANT 3", CERN DD/EE/84-1 (Revised), September 1987.

The GHEISHA program (H. Fesefeldt, RWTH Aachen Report PYTHIA 85/02 (1985)) is used to simulate hadronic interactions. 
[9] L3 Collab., O. Adriani et al., Physics Reports 236 (1993) 1.

[10] M. Biasini, Proceedings of the Third Workshop on Tau Lepton Physics, Montreux, Switzerland 1994, L. Rolandi edt., Nucl. Phys. B (Proc. Suppl.) 40 (1995) 331.

[11] Particle Data Group, R. M. Barnett et al., Phys. Rev. D 54 (1996) 1. 


\section{The L3 Collaboration:}

M.Acciarri ${ }^{28}$ O.Adriani ${ }^{17}$ M.Aguilar-Benitez ${ }^{27}$ S.Ahlen, ${ }^{11}$ B.Alpat,${ }^{35}$ J.Alcaraz ${ }^{27}$ G.Alemanni ${ }^{23}$ J.Allaby ${ }^{18}$ A.Aloisio ${ }^{30}$ G.Alverson ${ }^{12}$ M.G.Alviggi ${ }^{30}$ G.Ambrosi ${ }^{20}{ }^{20}$ H.Anderhub ${ }^{50}$ V.P.Andreev ${ }^{39}$ T.Angelescu ${ }^{13}$ F.Anselmo, D.Antreasyan, A.Arefiev ${ }^{29}$ T.Azemoon, T.Aziz ${ }^{10}$ P.Bagnaia ${ }^{38}$ L.Baksay ${ }^{45}$ R.C.Ball, ${ }^{3}$ S.Banerjee ${ }^{10}$ K.Banicz ${ }^{47}$ R.Barillère ${ }^{18}$ L.Barone ${ }^{38}$ P.Bartalini ${ }^{35}$ A.Baschirotto ${ }^{28}$ M.Basile, R.Battiston ${ }^{35}$ A.Bay ${ }^{23}$ F.Becattini, ${ }^{17}$ U.Becker ${ }^{16}$ F.Behner ${ }^{50}$ J.Berdugo ${ }^{27}$ P.Berges ${ }^{16}{ }^{3}$ B.Bertucci ${ }^{18}$ B.L.Betev ${ }^{50}$ S.Bhattacharya ${ }^{10}$ M.Biasini ${ }^{18}$ A.Biland ${ }^{50}$ G.M.Bilei ${ }^{35}$ J.J.Blaising, ${ }^{18}$ S.C.Blyth ${ }^{36}$ G.J.Bobbink, R.Bock, A.Böhm, ${ }^{1}$ B.Borgia ${ }^{38}$ A.Boucham, ${ }^{4}$ D.Bourilkov ${ }^{50}$ M.Bourquin ${ }^{20}$ D.Boutigny, J.G.Branson ${ }^{41}$ V.Brigljevic ${ }^{50}{ }^{4}$ I.C.Brock ${ }^{36}$ A.Buffini ${ }^{17}$ A.Buijs,${ }^{46}$ J.D.Burger,${ }^{16}$ W.J.Burger ${ }^{20}$ J.Busenitz ${ }^{45}$ A.Buytenhuijs ${ }^{32}$ X.D.Cai ${ }^{16}{ }^{6}$ M.Campanelli, ${ }^{50}$ M.Capell ${ }^{16}$ G.Cara Romeo, ${ }^{9}$ M.Caria ${ }^{35}$ G.Carlino, A.M.Cartacci ${ }^{17}{ }^{4}$ J.Casaus ${ }^{27}$ G.Castellini ${ }^{17}{ }^{17}$ F.Cavallari ${ }^{38}{ }^{38}$ N.Cavallo,${ }^{30}$ C.Cecchi ${ }^{20}$ M.Cerrada ${ }^{27}$ F.Cesaroni ${ }^{24}$ M.Chamizo, ${ }^{27}$ A.Chan ${ }^{52}$ Y.H.Chang, ${ }^{52}$ U.K.Chaturvedi ${ }^{19}$ M.Chemarin ${ }^{26}$ A.Chen ${ }^{52}$ G.Chen, G.M.Chen? H.F.Chen ${ }^{21}$ H.S.Chen? M.Chen ${ }^{16}$ G.Chiefari ${ }^{30}$ C.Y.Chien ${ }^{5}$ M.T.Choi ${ }^{44}{ }^{4}$ L.Cifarelli ${ }^{40}$ F.Cindolo ${ }^{9}$ C.Civinini ${ }^{17}$ I.Clare ${ }^{16}$ R.Clare ${ }^{16}$ H.O.Cohn ${ }^{33}$ G.Coignet ${ }^{4}$ A.P.Colijn, ${ }^{2}$ N.Colino ${ }^{27}$ S.Costantini ${ }^{38}$ F.Cotorobai ${ }^{13}$ B.de la Cruz ${ }^{27}$ A.Csilling ${ }^{14}$ T.S.Dai ${ }^{16}$ R.D'Alessandro ${ }^{17}$ R.de Asmundis,${ }^{30}$ H.De Boeck ${ }^{32}$ A.Degré ${ }^{4}$ K.Deiters ${ }^{48}$ P.Denes ${ }^{37}$ F.DeNotaristefani ${ }^{38}$ D.DiBitonto ${ }^{45}$ M.Diemoz ${ }^{38}$ D.van Dierendonck, F.Di Lodovico ${ }^{50}$ C.Dionisi ${ }^{38}$ M.Dittmar ${ }^{50}$ A.Dominguez ${ }^{41}$ A.Doria ${ }^{30}$ I.Dorne, M.T.Dova ${ }^{19, \sharp}$ E.Drago, ${ }^{30}$ D.Duchesneau, ${ }^{4}$ P.Duinker, I.Duran,${ }^{42}$ S.Dutta ${ }^{10}{ }^{\text {S.Easo }}{ }^{35}$ Yu.Efremenko ${ }^{33}$ H.El Mamouni ${ }^{26}$ A.Engler, ${ }^{36}$ F.J.Eppling, ${ }^{16}$ F.C.Erné, ${ }^{2}$ J.P.Ernenwein, ${ }^{26}$ P.Extermann, ${ }^{20}$ M.Fabre, ${ }^{48}$ R.Faccini ${ }^{38}$ S.Falciano ${ }^{38}$ A.Favara ${ }^{17}$ J.Fay ${ }^{26}$ O.Fedin ${ }^{39}$ M.Felcini ${ }^{50}$ B.Fenyi ${ }^{45}$ T.Ferguson ${ }^{36}$ D.Fernandez ${ }^{27}$ F.Ferroni ${ }^{38}{ }^{3}$ H.Fesefeldt ${ }^{1}$ E.Fiandrini,${ }^{35}$ J.H.Field ${ }^{20}$ F.Filthaut ${ }^{36}$ P.H.Fisher ${ }^{16}$ G.Forconi ${ }^{16}{ }^{2}$.Fredj ${ }^{20}$ K.Freudenreich ${ }^{50}$ C.Furetta ${ }^{28}$ Yu.Galaktionov ${ }^{29,16}$ S.N.Ganguli ${ }^{10}$ P.Garcia-Abia ${ }^{27}$ S.S.Gau, ${ }^{12}$ S.Gentile ${ }^{38}$ J.Gerald, ${ }^{5}$ N.Gheordanescu, ${ }^{13}$ S.Giagu ${ }^{38}$ S.Goldfarb ${ }^{23}$ J.Goldstein, ${ }^{11}$ Z.F.Gong, ${ }^{21}$ A.Gougas ${ }^{5}$ G.Gratta ${ }^{34}$ M.W.Gruenewald ${ }^{8}$ V.K.Gupta ${ }^{37}$ A.Gurtu,${ }^{10}$ L.J.Gutay,${ }^{47}$ K.Hangarter, ${ }^{1}$ B.Hartmann, ${ }^{1}$ A.Hasan, ${ }^{31}$ D.Hatzifotiadou, T.Hebbeker, A.Hervé, ${ }^{18}$ W.C.van Hoek ${ }^{32}$ H.Hofer,${ }^{50}$ H.Hoorani20 ${ }^{20}$ S.R.Hou ${ }^{52}$ G.Hu ${ }^{5}$ V.Innocente ${ }^{18}$ H.Janssen, ${ }^{4}$ B.N.Jin, L.W.Jones ${ }^{3}$ P.de Jong, ${ }^{16}$ I.Josa-Mutuberria ${ }^{27}{ }^{7}$ A.Kasser ${ }^{23}$ R.A.Khan ${ }^{19}$ Yu.Kamyshkov, ${ }^{33}$ P.Kapinos, ${ }^{49}$ J.S.Kapustinsky ${ }^{25}$ Y.Karyotakis, ${ }^{4}$ M.Kaur ${ }^{19,},{ }^{4}$ M.N.Kienzle-Focacci ${ }^{20}$ D.Kim ${ }^{5}$ J.K.Kim ${ }^{44}$ S.C.Kim ${ }^{44}$ Y.G.Kim ${ }_{1}^{44}$ W.W.Kinnison ${ }^{25}$ A.Kirkby ${ }^{34}$ D.Kirkby ${ }^{34}$ J.Kirkby ${ }^{18}$ D.Kiss ${ }^{14}$ W.Kittel ${ }^{32}$ A.Klimentov ${ }^{16,29}$ A.C.König, ${ }^{32}$ I.Korolko, ${ }^{29}$ V.Koutsenko ${ }^{16,29}$ R.W.Kraemer ${ }^{36}$ W.Krenz ${ }^{1}$ H.Kuijten ${ }^{32}$ A.Kunin ${ }^{16,29}$ P.Ladron de Guevara, ${ }^{27}$ G.Landi ${ }^{17}{ }^{7}$ C.Lapoint ${ }^{16}$ K.Lassila-Perini ${ }^{50}$ P.Laurikainen, ${ }^{22}$ M.Lebeau, ${ }^{18}$ A.Lebedev ${ }^{16}{ }^{6}$ P.Lebrun ${ }^{26}$ P.Lecomte, ${ }^{50}$ P.Lecoq ${ }^{18}$ P.Le Coultre ${ }^{50}$ J.S.Lee ${ }^{44}$ K.Y.Lee, ${ }^{44}$ C.Leggett ${ }^{3}$, J.M.Le Goff ${ }^{18}$ R.Leiste, ${ }^{49}$ E.Leonardi ${ }^{38}$ P.Levtchenko, ${ }^{39}$ C.Li ${ }^{21}$ E.Lieb ${ }^{49}$ W.T.Lin,${ }^{52}$ F.L.Linde, ${ }^{2,18}$ L.Lista ${ }^{30}$ Z.A.Liu, W.Lohmann, ${ }^{49}$ E.Longo ${ }^{38}$ W.Lu ${ }^{34}$ Y.S.Lu ${ }^{7}$ K.Lübelsmeyer ${ }^{1}$ C.Luci ${ }^{38}{ }^{2}$ D.Luckey ${ }^{16}$ L.Luminari ${ }^{38}$ W.Lustermann ${ }^{48}$ W.G.Ma ${ }^{21}$ M.Maity ${ }_{10}$ G.Majumder ${ }^{10}$ L.Malgeri ${ }^{38}$ A.Malinin ${ }^{29}{ }^{2}$ C.Maña ${ }^{27}$ S.Mangla ${ }_{1}^{10}$ P.Marchesini ${ }^{50}$ A.Marin, ${ }^{11}$ J.P.Martin ${ }^{26}$ F.Marzano ${ }^{38}$ G.G.G.Massaro, D.McNally ${ }^{18}$ S.Mele ${ }^{30}$ L.Merola,${ }^{30}$ M.Meschini ${ }^{17}$ W.J.Metzger, ${ }^{32}$ M.von der Mey ${ }^{1}$ Y.Mi ${ }^{23}$ A.Mihul ${ }_{1}^{13}$ A.J.W.van Mil ${ }^{32}$ G.Mirabelli, ${ }^{38}$ J.Mnich, ${ }^{18}$ P.Molnar, ${ }^{8}$ B.Monteleoni ${ }^{17}$ R.Moore, ${ }^{3}$ S.Morganti ${ }^{38}$ T.Moulik ${ }^{10}$ R.Mount, ${ }^{34}$ S.Müller ${ }^{1}$ F.Muheim ${ }^{20}$ E.Nagy ${ }_{14}^{14}$ S.Nahn ${ }^{16}$ M.Napolitano ${ }^{30}$ F.Nessi-Tedaldi ${ }^{50}$ H.Newman ${ }^{34}$ A.Nippe, H.Nowak, ${ }^{49}$ G.Organtini ${ }^{38}$ R.Ostonen ${ }^{22}$ D.Pandoulas, ${ }^{1}$ S.Paoletti ${ }^{38}$ P.Paolucci ${ }^{30}$ H.K.Park ${ }^{36}$ G.Pascale ${ }^{38}$ G.Passaleva ${ }^{17}$ S.Patricelli ${ }^{30}$ T.Paul ${ }^{12}$ M.Pauluzzi ${ }^{35}$ C.Paus,${ }^{1}$ F.Pauss ${ }^{50}$ D.Peach ${ }^{18}$ Y.J.Pei, ${ }^{1}$ S.Pensotti ${ }^{28}$ D.Perret-Gallix, ${ }^{4}$ S.Petrak, ${ }^{8}$ A.Pevsner, D.Piccolo, ${ }^{30}$ M.Pieri ${ }^{17}{ }^{1}$ J.C.Pinto ${ }^{36}$ P.A.Piroué, ${ }^{37}$ E.Pistolesi ${ }^{28}$ V.Plyaskin ${ }^{29}$ M.Poh ${ }^{50}{ }^{5}$ V.Pojidaev,${ }^{29,17}$ H.Postema ${ }^{16}$ N.Produit ${ }^{20}$ D.Prokofiev,${ }^{39}$ G.Rahal-Callot ${ }^{50}$ P.G.Rancoitaa ${ }^{28}$ M.Rattaggi ${ }^{28}$ G.Raven ${ }^{41}$ P.Razis,${ }^{31}$ K.Read ${ }^{33}$ D.Ren ${ }^{50}$ M.Rescigno ${ }^{38}$ S.Reucroft ${ }^{12}$ T.van Rhee,${ }^{46}$ S.Riemann ${ }^{49}$ B.C.Riemers, ${ }^{47}$ K.Riles ${ }^{3}$, O.Rind, ${ }^{3}$ S.Ro ${ }^{44}{ }^{4}$ A.Robohm ${ }^{50}{ }^{5}$ J.Rodin ${ }^{16}{ }^{1}$ F.J.Rodriguez ${ }^{27}$ B.P.Roe, ${ }^{3}$ S.Röhner, ${ }^{1}$ L.Romero ${ }^{27}$ S.Rosier-Lees, ${ }^{4}$ Ph.Rosselet ${ }^{23}$ W.van Rossum ${ }^{46}$ S.Roth ${ }_{1}^{1}$ J.A.Rubio ${ }^{18}$ H.Rykaczewski ${ }^{50}$ J.Salicio ${ }^{18}$ E.Sanchez ${ }^{27}$ A.Santocchia, ${ }^{35}$ M.E.Sarakinos, ${ }^{22}$ S.Sarkar ${ }^{10}$ M.Sassowsky ${ }^{1}$ G.Sauvage, ${ }^{4}$ C.Schäfer, V.Schegelsky ${ }^{39}$ S.Schmidt-Kaerst, D.Schmitz, P.Schmitz, M.Schneegans, ${ }^{4}$ B.Schoeneich, ${ }^{49}$ N.Scholz ${ }^{50}$ H.Schopper ${ }^{51}$ D.J.Schotanus ${ }^{32}$ J.Schwenke, G.Schwering, ${ }^{1}$ C.Sciacca ${ }^{30}$ D.Sciarrino, ${ }^{20}$ J.C.Sens, ${ }^{52}$ L.Servoli ${ }^{35}$ S.Shevchenko, ${ }^{34}$ N.Shivarov, ${ }^{43}$ V.Shoutko, ${ }^{29}$ J.Shukla ${ }^{25}$ E.Shumilov, ${ }^{29}$ A.Shvorob ${ }^{34}$ T.Siedenburg, D.Son ${ }^{44}$ A.Sopczak ${ }^{49}$ V.Soulimov, ${ }^{30}$ B.Smith ${ }^{16}$ P.Spillantini ${ }^{17}$ M.Steuer ${ }^{16}$ D.P.Stickland ${ }^{37}$ H.Stone ${ }^{37}$ B.Stoyanov ${ }^{43}$ A.Straessner, K.Strauch ${ }^{15}$ K.Sudhakar ${ }^{10}$ G.Sultanov, ${ }^{19}$ L.Z.Sun ${ }^{21}$ G.F.Susinno ${ }^{20}$ H.Suter ${ }^{50}$ J.D.Swain ${ }^{19}$ X.W.Tang, L.Tauscher, ${ }^{6}$ L.Taylor, ${ }^{12}$ Samuel C.C.Ting, ${ }^{16}$ S.M.Ting, ${ }^{16}$ F.Tonisch ${ }^{49}$ M.Tonutti, ${ }^{1}$ S.C.Tonwar ${ }^{10}$ J.Tóth ${ }^{14}{ }^{4}$ C.Tully ${ }^{37}$ H.Tuchscherer ${ }^{45}$ K.L.Tung ${ }^{7}$ Y.Uchida ${ }^{16}$ J.Ulbricht ${ }^{50}$ U.Uwer ${ }^{18}$ E.Valente ${ }^{38}$

R.T.Van de Walle, ${ }^{32}$ G.Vesztergombi ${ }^{14}$ I.Vetlitsky ${ }^{29}$ G.Viertel ${ }^{50}$ M.Vivargent ${ }^{4}$ R.Völkert ${ }^{49}$ H.Vogel ${ }^{36}{ }^{6}$ H.Vogt ${ }^{49}$ I.Vorobiev, ${ }^{29}$ A.A.Vorobyov ${ }^{39}$ A.Vorvolakos ${ }^{31}{ }^{1}$ M.Wadhwa ${ }^{6}$ W.Wallraff, ${ }^{1}$ J.C.Wang ${ }^{16}$ X.L.Wang ${ }^{21}$ Z.M.Wang ${ }^{21}$ A.Weber, ${ }^{1}$ F.Wittgenstein, ${ }^{18}$ S.X.Wu, ${ }^{19}$ S.Wynhoff, J.Xu ${ }^{11}{ }^{11}$ Z.Z.Xu, ${ }^{21}$ B.Z.Yang, ${ }^{21}$ C.G.Yang, X.Y.Yao, J.B.Ye, ${ }^{21}$

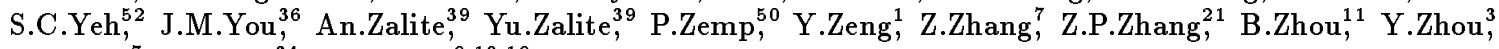
G.Y.Zhu, R.Y.Zhu, ${ }^{34}$ A.Zichichi: ${ }^{9,18,19}$ 
1 I. Physikalisches Institut, RWTH, D-52056 Aachen, FRG ${ }^{\S}$

III. Physikalisches Institut, RWTH, D-52056 Aachen, FRG ${ }^{\S}$

2 National Institute for High Energy Physics, NIKHEF, and University of Amsterdam, NL-1009 DB Amsterdam, The Netherlands

3 University of Michigan, Ann Arbor, MI 48109, USA

4 Laboratoire d'Annecy-le-Vieux de Physique des Particules, LAPP,IN2P3-CNRS, BP 110, F-74941 Annecy-le-Vieux CEDEX, France

5 Johns Hopkins University, Baltimore, MD 21218, USA

6 Institute of Physics, University of Basel, CH-4056 Basel, Switzerland

7 Institute of High Energy Physics, IHEP, 100039 Beijing, China ${ }^{\triangle}$

8 Humboldt University, D-10099 Berlin, FRG ${ }^{\S}$

9 INFN-Sezione di Bologna, I-40126 Bologna, Italy

10 Tata Institute of Fundamental Research, Bombay 400 005, India

11 Boston University, Boston, MA 02215, USA

12 Northeastern University, Boston, MA 02115, USA

13 Institute of Atomic Physics and University of Bucharest, R-76900 Bucharest, Romania

14 Central Research Institute for Physics of the Hungarian Academy of Sciences, H-1525 Budapest 114, Hungary ${ }^{\ddagger}$

15 Harvard University, Cambridge, MA 02139, USA

16 Massachusetts Institute of Technology, Cambridge, MA 02139, USA

17 INFN Sezione di Firenze and University of Florence, I-50125 Florence, Italy

18 European Laboratory for Particle Physics, CERN, CH-1211 Geneva 23, Switzerland

19 World Laboratory, FBLJA Project, CH-1211 Geneva 23, Switzerland

20 University of Geneva, CH-1211 Geneva 4, Switzerland

21 Chinese University of Science and Technology, USTC, Hefei, Anhui 230 029, China ${ }^{\triangle}$

22 SEFT, Research Institute for High Energy Physics, P.O. Box 9, SF-00014 Helsinki, Finland

23 University of Lausanne, CH-1015 Lausanne, Switzerland

24 INFN-Sezione di Lecce and Universitá Degli Studi di Lecce, I-73100 Lecce, Italy

25 Los Alamos National Laboratory, Los Alamos, NM 87544, USA

26 Institut de Physique Nucléaire de Lyon, IN2P3-CNRS,Université Claude Bernard, F-69622 Villeurbanne, France

27 Centro de Investigaciones Energeticas, Medioambientales y Tecnologicas, CIEMAT, E-28040 Madrid, Spainb

28 INFN-Sezione di Milano, I-20133 Milan, Italy

29 Institute of Theoretical and Experimental Physics, ITEP, Moscow, Russia

30 INFN-Sezione di Napoli and University of Naples, I-80125 Naples, Italy

31 Department of Natural Sciences, University of Cyprus, Nicosia, Cyprus

32 University of Nymegen and NIKHEF, NL-6525 ED Nymegen, The Netherlands

33 Oak Ridge National Laboratory, Oak Ridge, TN 37831, USA

34 California Institute of Technology, Pasadena, CA 91125, USA

35 INFN-Sezione di Perugia and Universitá Degli Studi di Perugia, I-06100 Perugia, Italy

36 Carnegie Mellon University, Pittsburgh, PA 15213, USA

37 Princeton University, Princeton, NJ 08544, USA

38 INFN-Sezione di Roma and University of Rome, "La Sapienza", I-00185 Rome, Italy

39 Nuclear Physics Institute, St. Petersburg, Russia

40 University and INFN, Salerno, I-84100 Salerno, Italy

41 University of California, San Diego, CA 92093, USA

42 Dept. de Fisica de Particulas Elementales, Univ. de Santiago, E-15706 Santiago de Compostela, Spain

43 Bulgarian Academy of Sciences, Central Laboratory of Mechatronics and Instrumentation, BU-1113 Sofia, Bulgaria

44 Center for High Energy Physics, Korea Advanced Inst. of Sciences and Technology, 305-701 Taejon, Republic of Korea

45 University of Alabama, Tuscaloosa, AL 35486, USA

46 Utrecht University and NIKHEF, NL-3584 CB Utrecht, The Netherlands

47 Purdue University, West Lafayette, IN 47907, USA

48 Paul Scherrer Institut, PSI, CH-5232 Villigen, Switzerland

49 DESY-Institut für Hochenergiephysik, D-15738 Zeuthen, FRG

50 Eidgenössische Technische Hochschule, ETH Zürich, CH-8093 Zürich, Switzerland

51 University of Hamburg, D-22761 Hamburg, FRG

52 High Energy Physics Group, Taiwan, China

$\S$ Supported by the German Bundesministerium für Bildung, Wissenschaft, Forschung und Technologie

$\doteqdot$ Supported by the Hungarian OTKA fund under contract number T14459.

b Supported also by the Comisión Interministerial de Ciencia y Technología

\# Also supported by CONICET and Universidad Nacional de La Plata, CC 67, 1900 La Plata, Argentina

$\diamond$ Also supported by Panjab University, Chandigarh-160014, India

$\triangle$ Supported by the National Natural Science Foundation of China. 